\title{
Expanding laparoscopic pancreaticoduodenectomy to pancreatic- head and periampullary malignancy: major findings based on systematic review and meta-analysis
}

\author{
Ke Chen', Xiao-long Liu', Yu Pan', Hendi Maher² and Xian-fa Wang ${ }^{1 *}$ (i)
}

\begin{abstract}
Background: Laparoscopic pancreaticoduodenectomy (LPD) remains to be established as a safe and effective alternative to open pancreaticoduodenectomy (OPD) for pancreatic-head and periampullary malignancy. The purpose of this meta-analysis was to compare LPD with OPD for these malignancies regarding short-term surgical and long-term survival outcomes.

Methods: A literature search was conducted before March 2018 to identify comparative studies in regard to outcomes of both LPD and OPD for the treatment of pancreatic-head and periampullary malignancies. Morbidity, postoperative pancreatic fistula (POPF), mortality, operative time, estimated blood loss, hospitalization, retrieved lymph nodes, and survival outcomes were compared.

Results: Among eleven identified studies, 1196 underwent LPD, and 8247 were operated through OPD. The pooled data showed that LPD was associated with less morbidity $(\mathrm{OR}=0.57,95 \% \mathrm{Cl}: 0.41 \sim 0.78, P<0.01)$, less blood loss $(\mathrm{WMD}=-372.96 \mathrm{ml}, 95 \% \mathrm{Cl},-507.83 \sim-238.09 \mathrm{ml}, P<0.01)$, shorter hospital stays (WMD $=-197.49 \mathrm{ml}, 95 \% \mathrm{Cl}$, - 304.62 - 90.37 ml, $P<0.01$ ), and comparable POPF (OR=0.85, 95\%Cl: 0.59 1.24, $P=0.40)$, and overall survival $(\mathrm{HR}=1.03,95 \% \mathrm{Cl}: 0.93 \sim 1.14, P=0.54)$ compared to $\mathrm{OPD}$. Operative time was longer in LPD (WMD = $87.68 \mathrm{~min}$; 95\% Cl: $27.05 \sim 148.32, P<0.01)$, whereas R0 rate tended to be higher in $\mathrm{LPD}(\mathrm{OR}=1.17 ; 95 \% \mathrm{Cl}: 1.00 \sim 1.37, P=0.05)$ and there tended to be more retrieved lymph nodes in $L P D(W M D=1.15,95 \% \mathrm{Cl}:-0.16 \sim 2.47, P=0.08)$, but these differences failed to reach statistical significance.

Conclusions: LPD can be performed as safe and effective as OPD for pancreatic-head and periampullary malignancy with respect to both surgical and oncological outcomes. LPD is associated with less intraoperative blood loss and postoperative morbidity and may serve as a promising alternative to OPD in selected individuals in the future.
\end{abstract}

Keywords: Laparoscopy, Pancreaticoduodenectomy, Adenocarcinoma, Morbidity, Meta-analysis

\footnotetext{
* Correspondence: 3195011@zju.edu.cn

${ }^{1}$ Department of General Surgery, Sir Run Run Shaw Hospital, School of

Medicine, Zhejiang University, 3 East Qingchun Road, Hangzhou 310016,

Zhejiang Province, China

Full list of author information is available at the end of the article
}

(c) The Author(s). 2018 Open Access This article is distributed under the terms of the Creative Commons Attribution 4.0 International License (http://creativecommons.org/licenses/by/4.0/), which permits unrestricted use, distribution, and reproduction in any medium, provided you give appropriate credit to the original author(s) and the source, provide a link to the Creative Commons license, and indicate if changes were made. The Creative Commons Public Domain Dedication waiver (http://creativecommons.org/publicdomain/zero/1.0/) applies to the data made available in this article, unless otherwise stated. 


\section{Background}

Pancreatic-head and periampullary malignancy mainly include pancreatic duct adenocarcinoma (PDAC) and periampullary adenocarcinoma (PAAC). PDAC causes a considerable amount of cancer-related death worldwide. In fact, PDAC is the fourth deadliest malignancy in developed countries and is predicted to become the second one within several years $[1,2]$. PAAC, defined as malignancy located in the distal common bile duct, ampulla of Vater or adjacent duodenum, are uncommon cancers compared to PDAC. However, despite its relatively higher resectability rates compared with PDAC, the long-term survival outcomes of PAAC patients are poor [3]. These cancers represent great challenges for healthcare providers and require a multidisciplinary approach in which pancreaticoduodenectomy (PD) with lymphadenectomy remains the primary curative treatment for patients without distant metastasis.

Minimally invasive surgery, typically characterized by laparoscopic approach, is one of the main surgical advances in the twenty-first Century [4]. It has been applied to complex pancreatic procedures including laparoscopic PD (LPD) for neoplasms on the pancreatic head and periampullar region or laparoscopic distal pancreatectomy (LDP) for these on the pancreatic body or tail. LDP represents a technique, which is technically less demanding, without any reconstruction, whereas LPD is a demanding procedure, which should be performed only in referral centers by experienced surgeons. Now, several meta-analyses have been published regarding LDP for malignancy treatment $[5,6]$, there was still no meta-analysis on the potential advantages and disadvantages of LPD for cancers. This meta-analysis proposed to dig deeper into the surgical and oncologic outcomes of patients who suffered from pancreatic-head and periampullary malignancy and underwent LPD versus open PD (OPD).

\section{Methods}

\section{Study selection}

We systematically searched the relevant literature using PubMed, Embase, Cochrane Library, and EBSCO for articles published up to March 2018 The search terms "minimally invasive", "laparoscopy", "Whipple", "pancreaticoduodenectomy", "pancreatic ductal adenocarcinoma", "pancreatic cancer", "ampullary cancer", "ampulla of Vater", and "periampullary neoplasm" were utilized. All eligible studies were retrieved, and their bibliographies were further checked for other potential publications. The language of the publications was confined to English.

\section{Inclusion criteria}

Publications included in this study were based on the following criteria: (1) compared LPD and OPD in patients suffering from pancreatic-head and periampullary malignancy
(PDAC and PAAC); (2) reported on at least one of the outcome measures mentioned below; and (3) if there was overlap between authors and/or institutions, the higher quality or more recent publication was selected.

\section{Data extraction}

Information was independently extracted from eligible studies by two authors (Chen K and Liu XL). The following information was collected: (1) Primary outcomes: morbidity, mortality, postoperative pancreatic fistula (POPF), margin status, retrieved lymph nodes (RLNs), and long-term survival. (2) Secondary outcomes: operative time, intraoperative blood loss, transfusion, and length of hospital stay. The postoperative morbidity was classified according to the Clavien-Dindo classification when possible [7]. POPF were diagnosed in accordance with the International Study Group for Pancreatic Fistula (ISGPF) criteria [8]. Clinically significant POPF was defined as ISGPF grade $\mathrm{B} / \mathrm{C}$. Resection margins were considered negative (R0) when no tumor was evident along the transection surface [9].

\section{Quality assessment}

The Newcastle-Ottawa Quality Assessment Scale (NOS) was used to evaluate the quality of non-randomized controlled trials (NRCTs). The quality of each study was scored by taking into account patient selection, comparability of the groups and assessment of the outcomes. The scale ranges from 0 to 9 stars: studies with a score $\geq 6$ could be deemed as methodologically sound. Randomized controlled trials (RCTs) were assessed by the Jadad scale. High quality RCTs get more than 2 out of a maximum possible score of 5 .

\section{Statistical analysis}

Statistical analysis was performed using the RevMan 5.1 software (Cochrane Collaboration, Oxford, UK). The odds ratio (OR) or weighted mean differences (WMD) with 95\% confidence intervals (CI) were calculated for dichotomous and continuous results, respectively. Medians were converted to means by the method introduced by Hozo et al. [10]. The hazard ratio (HR) was used as a summary statistic for long-term survival. The log HR and its standard error (SE) were analyzed directly if the studies reported the $\mathrm{HR}$ and 95\% CI. Otherwise, the log HR and its SE were estimated using the method described by Tierney et al. [11]. The fixed-effect model was firstly used for primary and secondary outcomes, and in case of significant heterogeneity $\left(I^{2}>50 \%\right.$ or $Q$ test $\left.P<0.05\right)$ the results were calculated using the random-effect model. Subgroup analysis was carried out according to the tumor primaries (PDAC or PAAC). Funnel plots were used to screen for publication bias based on the $\mathrm{R} 0$ rate. The 
statistical tests were two-sided, and $P<0.05$ was considered statistically significant.

\section{Results}

Study characteristics and quality of included studies

The search strategy initially generated 668 relevant clinical trials. Of these, articles that did not compare LPD with OPD were excluded based on their titles and abstracts. Thus, 86 articles were selected and a full examination of the text was conducted. A further 73 papers were excluded because the surgical indications of these studies were not restricted to pancreatic-head and periampullary malignancy. Another two publications were then excluded due to overlapping patient cohorts [12, 13]. Finally, a total of 9443 patients [LPD 1196 (12.7\%), OPD 8247 (87.3\%)] from 11 studies were included [14-24]. Figure 1 illustrates the selection process. Only one RCR was found [22]. The indication of six studies was PDAC [14, 16-20], whereas two studies applied LPD only to PAAC [15, 24], the remaining three researches reported both PDAC and PAAC [21-23]. Table 1 lists the characteristics of these studies and details of the enrolled participants. The RCT conducted by Palanivelu et al. received a Jadad score of 3 [22]. The quality evaluation using NOS showed that the included NRCTs were methodologically sound with four studies receiving 9 stars, five studies receiving 8 stars, and the remaining study receiving 7 stars (Table 1 ).

\section{Primary outcomes}

POPF was described in 8 studies [14, 15, 18, 19, 21-24]. Pooling data indicated no significant difference in terms of overall POPF rates between two groups $(\mathrm{OR}=0.85$, 95\%CI: $0.59 \sim 1.24, P=0.40$ ) (Fig. 2 ), as well as the clinically significant POPF rates $(\mathrm{OR}=0.86,95 \% \mathrm{CI}$ : $0.53 \sim 1.41$,
$P=0.56)$. The morbidity was available from 7 studies $[14,15,18,19,21-23]$. The pooled data indicated that the overall postoperative morbidity was significantly decreased in LPD (OR $=0.57,95 \% \mathrm{CI}: 0.41 \sim 0.78, P<0.01)$ (Fig. 3). Eight studies recorded postoperative mortality $[14,15,19-24]$. Mortality was similar in LPD and OPD for malignancies $(\mathrm{OR}=0.88,95 \% \mathrm{CI}$ : $0.64 \sim 1.20, P=0.41$ ) (Fig. 4). Negative margin (R0) rate was reported in all studies. The difference in R0 rate was not significant in pooling results, but had a tendency to be higher in the LPD group than in the OPD group $(\mathrm{OR}=1.17,95 \% \mathrm{CI}$ : $1.00 \sim 1.37, P=0.05$ ) (Fig. 5). The funnel plot for studies reporting the ORs of R0 was used to detect publication bias. Visual inspection of the funnel plot revealed symmetry, indicating no serious publication bias, as illustrated in Fig. 6. The RLNs were pooled from 10 studies [14-17, 19-24]. Pooled results revealed a tendency of more RLNs in the LPD group than in the OPD group with a marginal difference $(\mathrm{WMD}=1.15,95 \% \mathrm{CI}$ : $-0.16 \sim 2.47, P=0.08$ ) (Fig. 7). Six studies reported survival outcomes. The overall survival rate was not found to be different among the two groups $(\mathrm{HR}=1.03$, 95\%CI: 0.93 1.14, $P=0.54)$ (Fig. 8). The primary outcomes of the quantitative meta-analysis were summarized in Table 2.

\section{Secondary outcomes}

Five studies reported operative time [14, 19, 22-24]. The operative time in LPD group was longer than that in OPD group (WMD = $87.68 \mathrm{~min}, 95 \% \mathrm{CI}$ : 27.05 148.32, $P<0.01$ ). Also five studies reported blood loss [14, 19, 22-24]. The estimated blood loss was significantly reduced in LPD group (WMD = - $197.49 \mathrm{ml}, 95 \% \mathrm{CI},-304.62 \sim-90.37 \mathrm{ml}$, $P<0.01)$. A similar result was achieved in the field of blood transfusions $(\mathrm{OR}=0.64,95 \% \mathrm{CI}: 0.50 \sim 0.84, P<0.01)$. Nine

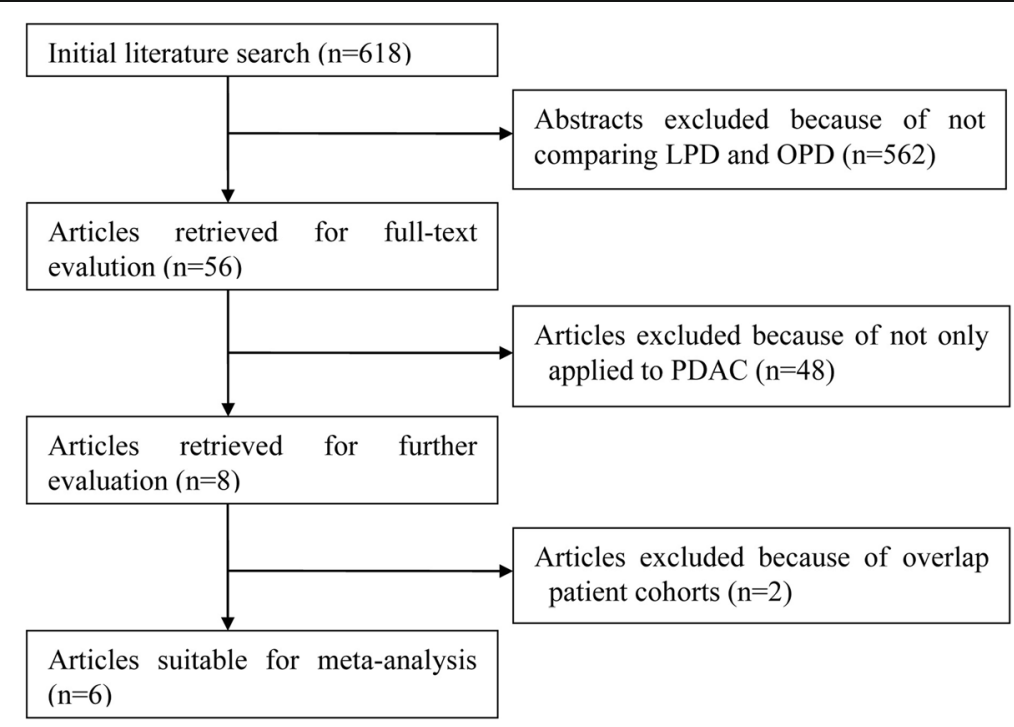

Fig. 1 Flow chart of literature search strategies 
Table 1 Summary of studies included in the meta-analysis

\begin{tabular}{|c|c|c|c|c|c|c|c|c|c|c|c|c|c|}
\hline \multirow[t]{2}{*}{ Author } & \multirow[t]{2}{*}{ Region } & \multirow[t]{2}{*}{ Design } & \multirow[t]{2}{*}{ Year } & \multirow[t]{2}{*}{ Study Period } & \multicolumn{2}{|c|}{ Sample size } & \multirow[t]{2}{*}{ Indications } & \multirow{2}{*}{$\begin{array}{l}\text { Conversion } \\
\mathrm{n}(\%)\end{array}$} & \multirow[t]{2}{*}{ ITT } & \multirow[t]{2}{*}{ ISGPF } & \multirow[t]{2}{*}{ Clavien-Dindo } & \multirow[t]{2}{*}{ Mortality } & \multirow{2}{*}{$\begin{array}{l}\text { Quality } \\
\text { scores }\end{array}$} \\
\hline & & & & & LPD & OPD & & & & & & & \\
\hline Croome & USA & $\mathrm{OCS}(\mathrm{P}, \mathrm{S})$ & 2014 & 2008-2013 & 108 & 214 & PDAC & $7(6.5)$ & Yes & Yes & Yes & $30 d$ & 8 \\
\hline Hakeem & UK & $\operatorname{OCS}(R, S)$ & 2014 & 2005-2009 & 12 & 12 & PAAC & NR & NR & NR & Yes & $30 d$ & 9 \\
\hline Chen & China & OCS $(P, S)$ & 2015 & 2010-2013 & 19 & 38 & PDAC & $1(5.3)$ & Yes & Yes & Yes & NR & 8 \\
\hline Song & Korea & OCS $(R, S)$ & 2015 & 2007-2012 & 11 & 261 & PDAC & NR & No & Yes & Yes & $30 d$ & 8 \\
\hline Dokmak & France & OCS $(P, S)$ & 2015 & $2011-2014$ & 15 & 14 & PDAC & NR & Yes & Yes & Yes & $90 d$ & 7 \\
\hline Stauffer & USA & OCS $(P, S)$ & 2017 & 1995-2014 & 58 & 193 & PDAC & $14(24.1)$ & Yes & Yes & Yes & $90 d$ & 8 \\
\hline Kantor & USA & OCS (R,M) & 2017 & 2010-2013 & 828 & 7385 & PDAC & $E$ & NR & NR & NR & $90 d$ & 8 \\
\hline Conrad & USA & $\mathrm{OCS}(P, S)$ & 2017 & 2000-2010 & 40 & 25 & Mixed & $9(18.4)^{a}$ & No & Yes & Yes & $90 d$ & 9 \\
\hline Palanivelu & India & RCT & 2017 & 2013-2015 & 32 & 32 & Mixed & $1(3.1)$ & Yes & Yes & Yes & $90 d$ & $3^{\mathrm{a}}$ \\
\hline Khaled & UK & $\operatorname{OCS}(R, S)$ & 2017 & 2002-2015 & 15 & 15 & Mixed & $1(6.7)$ & Yes & Yes & Yes & $30 d$ & 9 \\
\hline Meng & China & OCS (R,S) & 2018 & 2010-2015 & 58 & 58 & PAAC & NR & NR & Yes & Yes & $30 d$ & 9 \\
\hline
\end{tabular}

OCS observational clinical study, $R C T$ randomized controlled trial, $P$ prospectively collected data, $R$ retrospectively collected data, $M$ muti-centers, $S$ single center, $D P$ distal pancreatectomy, $P D$ pancreatoduodenectomy, $L$ laparoscopy, $O$ open, ITT intention-to-treat analysis, ISGPF international study group of pancreatic fistula, $P J$ pancreaticojejunostomy, DTM duct-to-mucosa, $E$ exclude, $N R$ not reported

a Jadad score

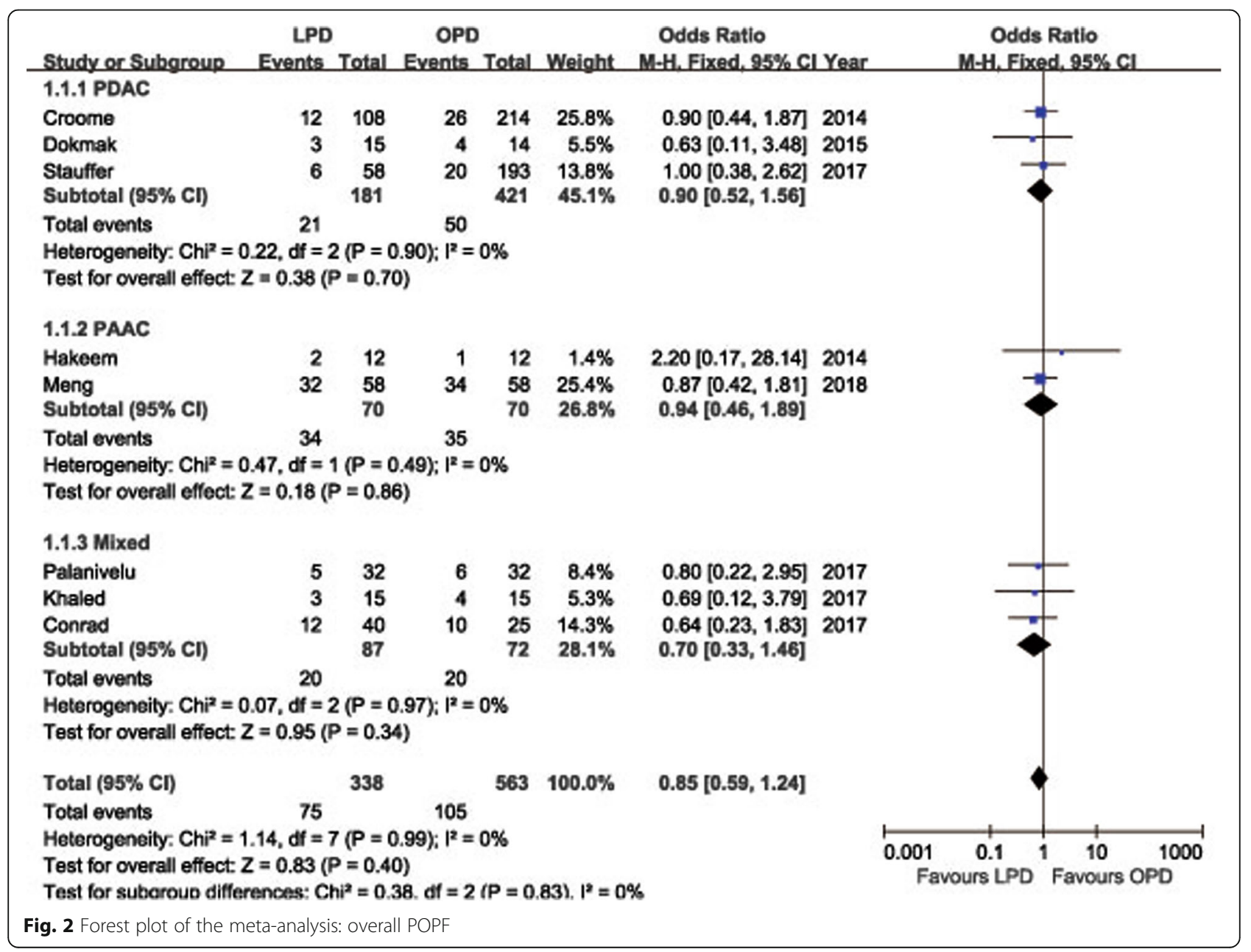




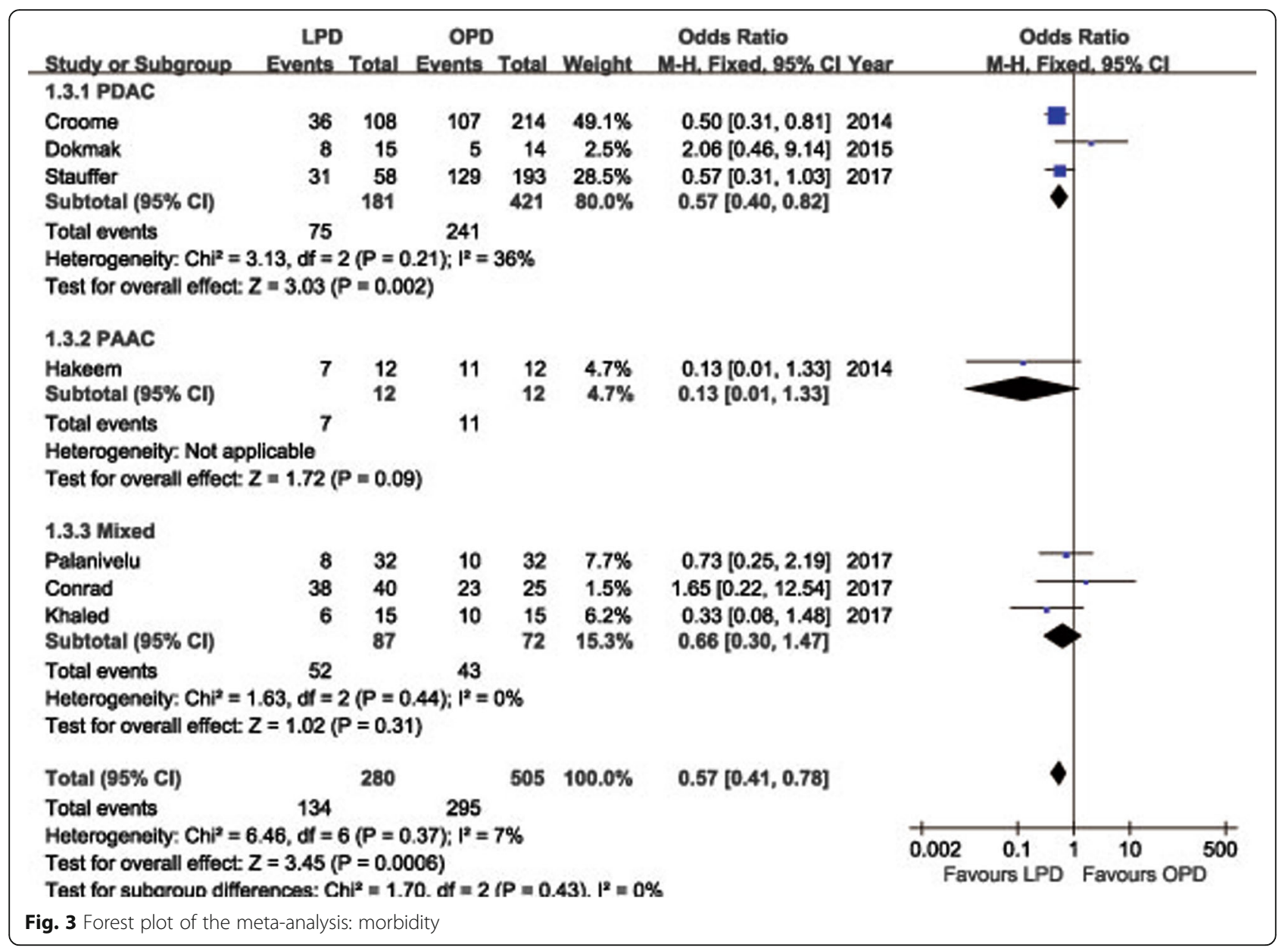

studies reported the length of hospital stay [14, 15, 18-24]. The pooled data indicated a comparable length of hospital stay between groups (WMD $=-1.07,95 \% \mathrm{CI},-3.05 \sim-0.92$, $P=0.29)$. Tumor size was available except in one study [20]. The tumor size of OPD was larger than that of LPD (WMD $=-0.16,95 \% \mathrm{CI},-0.31 \sim-0.02, P=0.03)$. The secondary outcomes of the quantitative meta-analysis are outlined in Table 2.

\section{Sensitivity analysis}

One retrospectively muti-institutional study conducted by Kantor et al. [20], in which only PDAC were included, provided the vast majority of cases. The study offered outcomes of mortality, R0 rate, RLNs, overall survival, and length of hospital stay. We investigated the influence of this study on the overall estimated risk by sequentially removing it from the pooled outcomes. We found only the pooling data of R0 rate changed from marginal difference $(\mathrm{OR}=1.17,95 \% \mathrm{CI}: 1.00 \sim 1.37, P=0.05)$ to no significant difference $(\mathrm{OR}=1.28,95 \% \mathrm{CI}$ : $0.89 \sim 1.84$, $P=0.19$ ), whereas the main results presented no obvious changes.

\section{Discussion}

Minimally invasive PD (MIPD), a laparoscopic surgery, was first described by Gagner and Pompin in 1994, and there has been a recent surge of interest for this demanding technique $[25,26]$. PD is a complex procedure and the advantages of minimally invasive approaches used to be closely scrutinized. Although several meta-analyses have confirmed the advantages of MIPD over open surgery, there has been no analysis restricted to malignancy. Moreover, effects of oncologic results were not well evaluated due to insufficient data. In Table 3, we present several previous meta-analyses comparing MIPD to OPD for benign and malignant periampullary disease [27-34].

This meta-analysis selected and summarized the available literature that compared the short-term surgical and long-term survival results of LPD and OPD for malignant periampullary disease. To the best of our knowledge, this is the first meta-analysis comparing LPD versus OPD for the treatment of pancreatic-head and periampullary cancer. No statistically significant differences were identified between the two groups regarding POPF, mortality, overall survival rate, and hospital stay. 


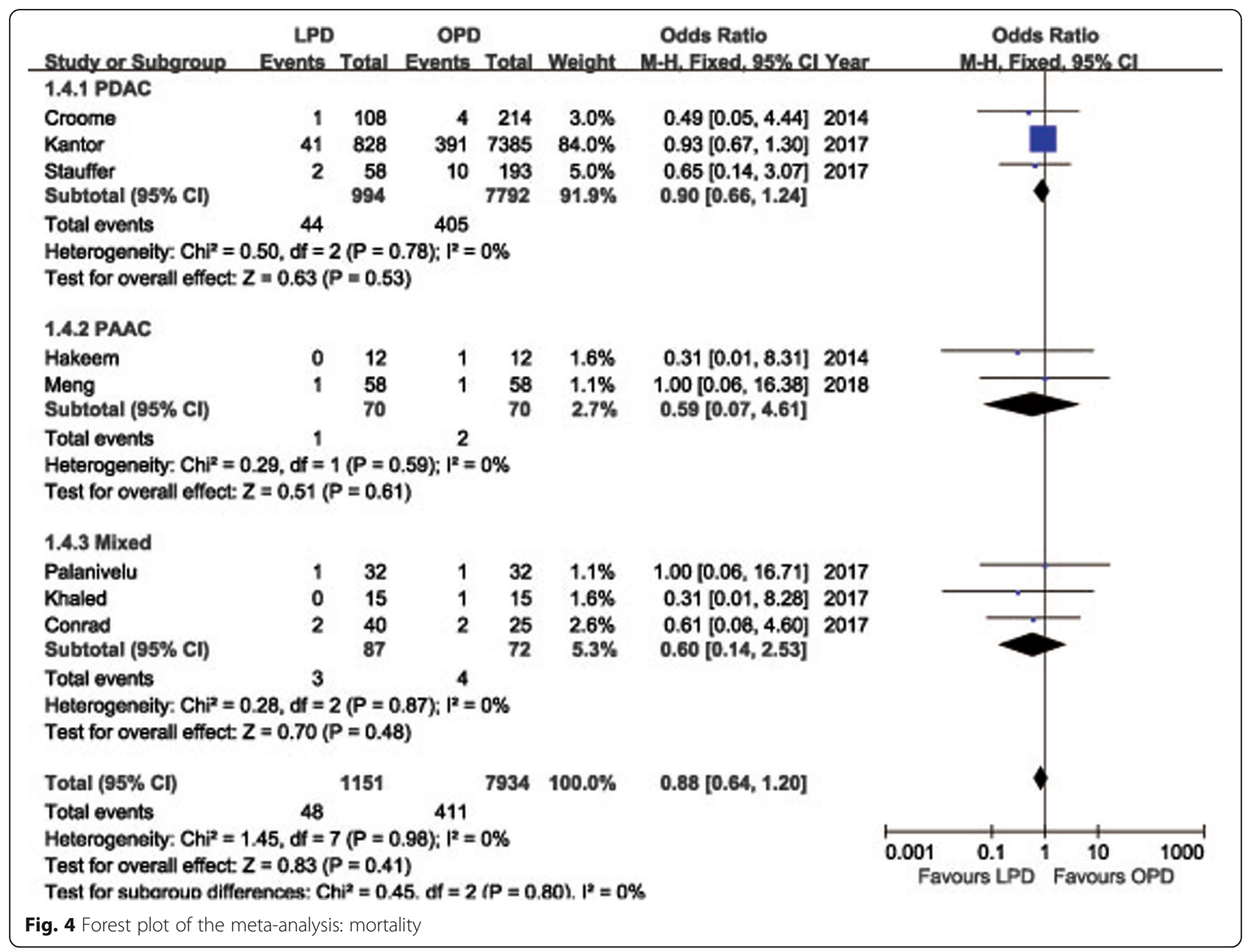

Operative time was significantly longer in the LPD group. The differences of R0 rate, and RLNs did not reach statistical significance, but tended to be superior in the LPD group. Moreover, LPD exhibited statistically significant benefits in terms of blood loss, and overall postoperative complications.

The progress of LPD was slow due to the threatening complication of POPF. High rates ranging from 4 to $33 \%$ for conventional open surgery were previously reported for both benign and malignant lesions $[35,36]$. POPF rates range from 11.8 to $55.2 \%$ in LPD for malignancy as reported seemed to somewhat higher than the results above (Table 4). It was also reported that LPD for resection of periampullary tumors was associated with higher morbidity, mainly due to severe POPF [18]. However, our pooling data demonstrated comparable rates, regardless of overall POPFs or clinically significant POPFs between LPD and OPD for malignancy. The approaches which could reduce POPFs also can be completed under laparoscope [37]. The end-to-side, duct-to-mucosa pancreaticojejunostomy (PJ) was now the most commonly performed pancreatic anastomosis approach under laparoscopy just as the conventional open approach [38]. Importantly, our results have shown improved overall morbidity of LPD. Since surgical complications, mainly POPFs, were comparable between the two groups because of the same organ and lymphatic resection area of LPD and OPD, the reduced overall complications may be the result of fewer medical complications. PD involves multiple systems and the complexity of performing three anastomoses can result in enormous surgical trauma, which would result in high risks of medical complications. Pulmonary, cardiovascular, and cerebrovascular morbidities were the most frequent systemic complications in major abdominal surgery. Less bleeding and use of transfusion contribute to preserve stable pulmonary and cardiovascular functions [39]. In addition, less pain and earlier ambulation allows patients to restore physiological homeostasis.

The pooling results show that LPD is linked with a tendency of lower positive margin rate $(P=0.05)$ and more retrieved lymph nodes $(P=0.08)$, two of the oncologic outcomes. Appropriate lymphadenectomy is crucial because elimination of a sufficient quantity of lymphadens could help to strengthen the staging accuracy and 


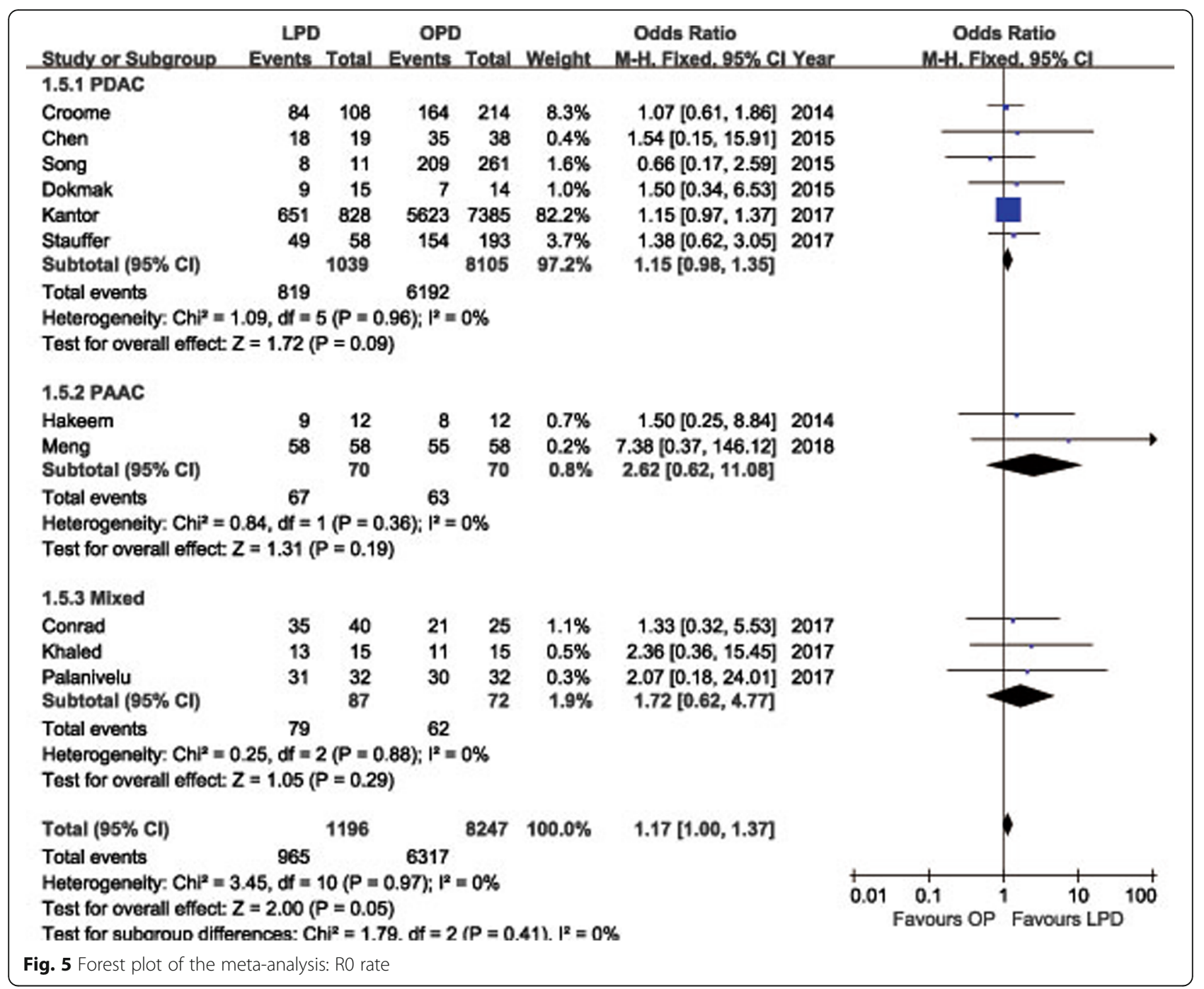

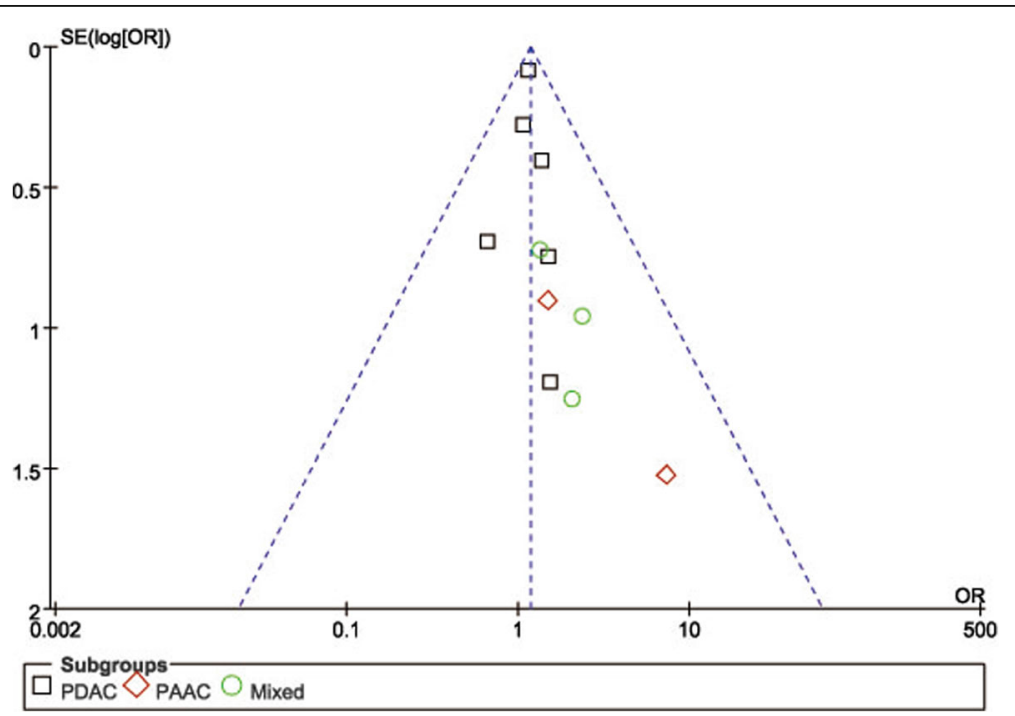

Fig. 6 Funnel plots of the RO rate 


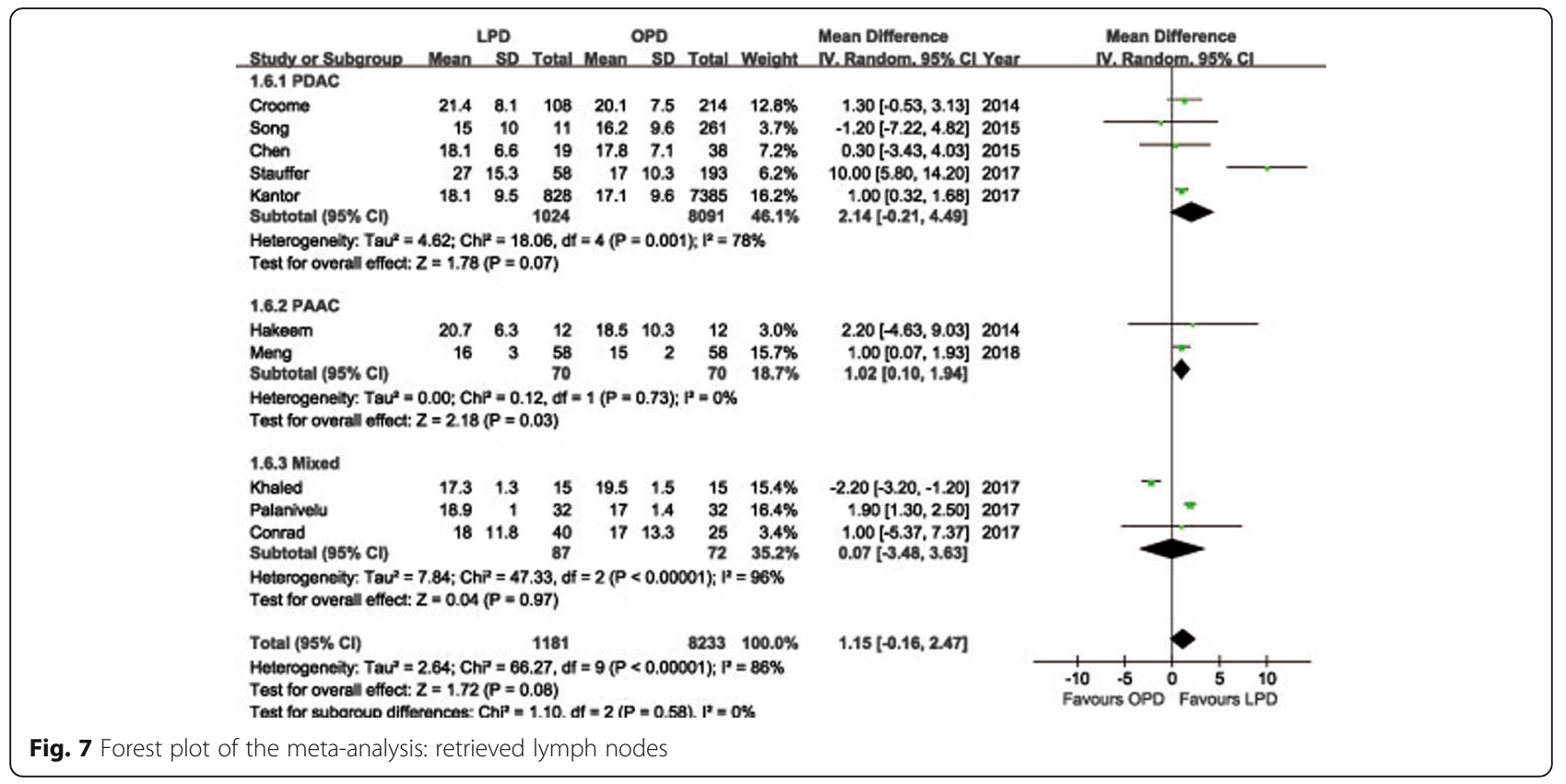

\begin{tabular}{|c|c|c|c|c|}
\hline Study or Subgroup & log[Risk Ratio] SE & Weight & $\begin{array}{l}\text { Risk Ratio } \\
\text { IV. Fixed. } 95 \% \text { CI Year }\end{array}$ & $\begin{array}{c}\text { Risk Ratio } \\
\text { IV. Fixed.95\% cl }\end{array}$ \\
\hline \multicolumn{5}{|l|}{ 1.7.1 PDAC } \\
\hline Croome & $0.26 \quad 0.17$ & $8.8 \%$ & $1.30[0.93,1.81] 2014$ & 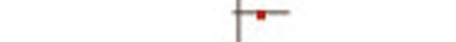 \\
\hline Chen & 0.090 .15 & $11.3 \%$ & $1.09[0.82,1.47] 2015$ & \\
\hline Stauffer & $-0.26 \quad 0.2$ & $6.4 \%$ & $0.77[0.52,1.14] 2017$ & \\
\hline $\begin{array}{l}\text { Kantor } \\
\text { Subtotal }(95 \% \mathrm{Cl})\end{array}$ & 0.020 .06 & $\begin{array}{l}70.9 \% \\
97.4 \%\end{array}$ & $\begin{array}{l}1.02[0.91,1.15] 2017 \\
1.03[0.93,1.14]\end{array}$ & \\
\hline \multicolumn{5}{|c|}{$\begin{array}{l}\text { Heterogeneity: } \text { ChP }^{2}=4.12, \mathrm{df}=3(P=0.25) ; 1^{2}=27 \% \\
\text { Test for overall effect: } Z=0.62(P=0.54)\end{array}$} \\
\hline \multicolumn{5}{|l|}{ 1.7.2 PAAC } \\
\hline $\begin{array}{l}\text { Hakeem } \\
\text { Subtotal }(95 \% \mathrm{Cl})\end{array}$ & $-0.76 \quad 0.66$ & $\begin{array}{l}0.6 \% \\
0.6 \%\end{array}$ & $\begin{array}{l}0.47[0.13,1.71] 2014 \\
0.47[0.13,1.71]\end{array}$ & \\
\hline \multicolumn{5}{|c|}{$\begin{array}{l}\text { Heterogeneity: Not applicable } \\
\text { Test for overall effect: } Z=1.15(P=0.25)\end{array}$} \\
\hline \multicolumn{5}{|l|}{ 1.7.3 Mixed } \\
\hline $\begin{array}{l}\text { Conrad } \\
\text { Subtotal }(95 \% \mathrm{Cl})\end{array}$ & -0.20 .36 & $\begin{array}{l}2.0 \% \\
2.0 \%\end{array}$ & $\begin{array}{l}0.82[0.40,1.66] 2017 \\
0.82[0.40,1.66]\end{array}$ & \\
\hline \multicolumn{5}{|c|}{$\begin{array}{l}\text { Heterogeneity: Not applicable } \\
\text { Test for overall effect: } Z=0.56(P=0.58)\end{array}$} \\
\hline Total $(95 \% \mathrm{Cl})$ & & $100.0 \%$ & $1.02[0.93,1.13]$ & \\
\hline \multicolumn{4}{|c|}{$\begin{array}{l}\text { Heterogeneity: } \text { Chi }^{2}=5.94, d f=5(P=0.31) ; I^{2}=16 \% \\
\text { Test for overall effect: } Z=0.44(P=0.66) \\
\text { Test for suborouo differences: } \mathrm{Ch}^{2}=1.82 . \mathrm{df}=\mathbf{2}(\mathrm{P}=0.40) . P=0 \%\end{array}$} & 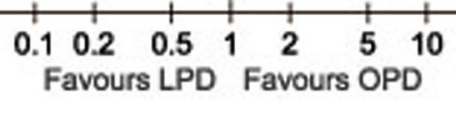 \\
\hline \multicolumn{5}{|c|}{ ig. 8 Forest plot of the meta-analysis: overall survival rate } \\
\hline
\end{tabular}


Table 2 Results of the meta-analysis

\begin{tabular}{|c|c|c|c|c|c|c|c|c|}
\hline \multirow[t]{2}{*}{ Outcomes } & \multirow{2}{*}{$\begin{array}{l}\text { No. } \\
\text { Studies }\end{array}$} & \multicolumn{2}{|c|}{ Sample size } & \multirow{2}{*}{$\begin{array}{l}\text { Heterogeneity } \\
\left(P, I^{2}\right)\end{array}$} & \multirow[t]{2}{*}{ Model } & \multirow{2}{*}{$\begin{array}{l}\text { Overall effect } \\
\text { size }\end{array}$} & \multirow{2}{*}{$\begin{array}{l}95 \% \mathrm{Cl} \text { of } \\
\text { overall effect }\end{array}$} & \multirow[t]{2}{*}{$P$} \\
\hline & & LPD & OPD & & & & & \\
\hline \multicolumn{9}{|l|}{ Primary Outcomes } \\
\hline POPF & 8 & 338 & 563 & $0.99,0 \%$ & $\mathrm{~F}$ & $\mathrm{OR}=0.85$ & $0.59 \sim 1.24$ & 0.40 \\
\hline Significant POPF & 5 & 271 & 512 & $0.96,0 \%$ & $\mathrm{~F}$ & $\mathrm{OR}=0.86$ & $0.53 \sim 1.41$ & 0.56 \\
\hline Morbidity & 7 & 280 & 505 & $0.37,7 \%$ & $\mathrm{~F}$ & $\mathrm{OR}=0.57$ & $0.41 \sim 0.78$ & $<0.01$ \\
\hline Mortality & 8 & 1151 & 7934 & $0.98,0 \%$ & $\mathrm{~F}$ & $\mathrm{OR}=0.88$ & $0.64 \sim 1.20$ & 0.41 \\
\hline RO rate & 11 & 1196 & 8247 & $0.97,0 \%$ & $\mathrm{~F}$ & $\mathrm{OR}=1.17$ & $1.00 \sim 1.37$ & 0.05 \\
\hline Retrieved lymph nodes & 10 & 1181 & 8233 & $<0.01,86 \%$ & $\mathrm{R}$ & WMD = 1.15 & $-0.16 \sim 2.47$ & 0.08 \\
\hline Overall survival & 6 & 1065 & 7867 & $0.31,16 \%$ & $\mathrm{R}$ & $\mathrm{HR}=1.02$ & $0.93 \sim 1.13$ & 0.66 \\
\hline \multicolumn{9}{|l|}{ Secondary Outcomes } \\
\hline Operation time (min) & 5 & 271 & 512 & $<0.01,99 \%$ & $\mathrm{R}$ & WMD $=87.68$ & $27.05 \sim 148.32$ & $<0.01$ \\
\hline Blood loss (mL) & 5 & 271 & 512 & $<0.01,96 \%$ & $\mathrm{R}$ & WMD = -197.49 & $-304.62 \sim-90.37$ & $<0.01$ \\
\hline Transfusion & 5 & 296 & 522 & $0.36,7 \%$ & $\mathrm{~F}$ & $\mathrm{OR}=0.64$ & $0.50 \sim 0.84$ & $<0.01$ \\
\hline Hospital stay (days) & 9 & 1166 & 7948 & $<0.01,85 \%$ & $\mathrm{R}$ & WMD $=-1.07$ & $-3.05 \sim 0.92$ & 0.29 \\
\hline Tumor size & 10 & 368 & 862 & $0.46,0 \%$ & $\mathrm{~F}$ & WMD $=-0.16$ & $-0.31 \sim-0.02$ & 0.03 \\
\hline
\end{tabular}

regional tumor control. In addition, curative R0 resection is referred as the most important factor determining, which is deemed the only chance to survival [40], and the prognostic validity of margin status may be primarily confined to pancreatic head cancers rather than neoplasms in body or tail [41]. Elaborate manipulation and better visualization of critical anatomy could explain our outcomes. However, as our results also indicated a shorter tumor size in LPD group, some researches may include LPD cases of small, easily resectable tumors that would be partial to LPD, the benefit of LPD for margin status and lymph nodes harvesting cannot be confirmed, but at least not inferior to open surgery. Long-term survival rate is crucial the outcome for evaluating surgical interventions in oncological therapy. Periampullary cancer, especially PDAC, has significantly more aggressive inherent tumor biology, for which there are hardly any effect of adjuvant therapy $[42,43]$. Large series on PDAC reported 5-year survival rates only around 20\% [44, 45], and estimated at only $20-50 \%$ for PAAC $[3,46]$. None of the previous studies on LPD identify survival advantages of the laparoscopic approach, and our analysis

Table 3 Previous meta-analyses comparing MIPD with OPD for benign and malignant periampullary disease

\begin{tabular}{|c|c|c|c|c|c|c|c|c|c|}
\hline Variables & Correa & Nigri & Lei & Qin & de Rooij & Zhang & Peng & Shin $^{b}$ & Shin $^{b}$ \\
\hline Year & 2014 & 2014 & 2014 & 2014 & 2016 & 2016 & 2017 & 2017 & 2017 \\
\hline Included studies & 6 & 8 & 9 & 11 & 19 & 22 & 9 & 8 & 5 \\
\hline Minimally invasive method & MIPD & MIPD & MIPD & MIPD & MIPD & MIPD & RPD & LPD & RPD \\
\hline Total MIPD numbers & 169 & 204 & 209 & 327 & 710 & 1018 & 245 & 450 & 160 \\
\hline POPF & NS & NS & NS & NS & NS & NS & NS & NS & NS \\
\hline Morbidity & NS & NS & NS & $N S^{a}$ & N/A & NS & Favor RPD & NS & $N S^{a}$ \\
\hline Mortality & $\mathrm{N} / \mathrm{A}$ & NS & NS & NS & NS & NS & NS & $\mathrm{N} / \mathrm{A}$ & $\mathrm{N} / \mathrm{A}$ \\
\hline R0 rate & Favor MIPD & NS & Favor MIPD & $N S^{a}$ & Favor MIPD & Favor MIPD & Favor RPD & NS & NS \\
\hline Retrieved lymph nodes & Favor MIPD & $N S^{a}$ & NS & NS & NS & $N S^{a}$ & NS & NS & NS \\
\hline Survival & $\mathrm{N} / \mathrm{A}$ & $\mathrm{N} / \mathrm{A}$ & N/A & N/A & $\mathrm{N} / \mathrm{A}$ & $\mathrm{N} / \mathrm{A}$ & $\mathrm{N} / \mathrm{A}$ & N/A & N/A \\
\hline Operation time & Favor OPD & Favor OPD & Favor OPD & Favor OPD & Favor OPD & Favor OPD & NS & Favor OPD & Favor OPD \\
\hline Blood loss & Favor MIPD & Favor MIPD & Favor MIPD & Favor MIPD & Favor MIPD & Favor MIPD & $\mathrm{N} / \mathrm{A}$ & NS & Favor RPD \\
\hline Transfusion & $\mathrm{N} / \mathrm{A}$ & NS & Favor MIPD & $\mathrm{N} / \mathrm{A}$ & $\mathrm{N} / \mathrm{A}$ & Favor MIPD & $\mathrm{N} / \mathrm{A}$ & $\mathrm{N} / \mathrm{A}$ & $\mathrm{N} / \mathrm{A}$ \\
\hline Hospital stay (days) & Favor MIPD & Favor MIPD & Favor MIPD & Favor MIPD & Favor MIPD & Favor MIPD & Favor RPD & Favor LPD & Favor RPD \\
\hline
\end{tabular}

NS not significant, $N / A$ not available

${ }^{a}$ not significant but tended to favor MIPD

b one separate study 
Table 4 Studies on LPD for pancreatic cancer

\begin{tabular}{|c|c|c|c|c|c|c|c|c|c|c|c|}
\hline & Croome & Hakeem & Chen & Song & Dokmak & Stauffer & Kantor & Conrad & Palanivelu & Khaled & Meng \\
\hline Age (years) & $66.6 \pm 9.6$ & $67.0 \pm 10.2$ & - & $68.1 \pm 7$ & - & $69.9(40.6-84.8)$ & $65.9 \pm 10.7$ & $68(45-83)$ & $57.8 \pm 2.0$ & $\begin{array}{l}65 \\
(35-78)\end{array}$ & $60.0 \pm 9.1$ \\
\hline $\operatorname{Sex}(M / F)$ & $57 / 51$ & $8 / 4$ & - & - & - & $32 / 26$ & - & $26 / 14$ & $18 / 14$ & $8 / 7$ & $32 / 26$ \\
\hline BMI & $27.4 \pm 5.4$ & $25.8 \pm 3.7$ & - & - & - & $25.9(17.7-49.6)$ & - & $\begin{array}{l}23.9 \\
(14.9-34.1)\end{array}$ & $24.9 \pm 0.7$ & $\begin{array}{l}23.4 \\
(18-26)\end{array}$ & $22.3 \pm 3.0$ \\
\hline $\begin{array}{l}\text { Tumor size } \\
(\mathrm{cm})\end{array}$ & $3.3 \pm 1.0$ & $2.0 \pm 1.0$ & $3.0 \pm 0.9$ & $2.8 \pm 0.6$ & $2.4(1.5-4)$ & $2.5(0.3-10.0)$ & - & $\begin{array}{l}2.5 \\
(0.3-8.0)\end{array}$ & $3.3 \pm 0.7$ & $\begin{array}{l}2.0 \\
(0.7-8.0)\end{array}$ & $\begin{array}{l}1.9 \\
(1.5-2.6)\end{array}$ \\
\hline Retrieved LNs & $21.4 \pm 8.1$ & $20.7 \pm 6.3$ & $18.1 \pm 6.6$ & $15 \pm 10$ & $20(8-59)$ & $27(9-70)$ & $18.1 \pm 9.5$ & $18(6-53)$ & $18.9 \pm 1.0$ & $\begin{array}{l}18 \\
(14-19)\end{array}$ & $16(15-18)$ \\
\hline RO rate & $77.8 \%$ & $75.0 \%$ & $94.7 \%$ & $72.7 \%$ & $60 \%$ & $84.5 \%$ & $79.1 \%$ & $87.5 \%$ & $96.9 \%$ & $86.7 \%$ & $100 \%$ \\
\hline $\begin{array}{l}\text { Operative } \\
\text { time (min) }\end{array}$ & $379.4 \pm 93.5$ & - & - & - & - & $518(313-761)$ & - & - & $359 \pm 14$ & $\begin{array}{l}470 \\
(280-660)\end{array}$ & $\begin{array}{l}475 \\
(420-546)\end{array}$ \\
\hline $\begin{array}{l}\text { Blood loss } \\
(\mathrm{mL})\end{array}$ & $492.4 \pm 519.3$ & - & - & - & - & $250(50-8500)$ & - & - & $250 \pm 22$ & $\begin{array}{l}300 \\
(50-600)\end{array}$ & $\begin{array}{l}200 \\
(100-325)\end{array}$ \\
\hline POPF & - & $16.7 \%$ & - & - & $20 \%$ & $11.8 \%$ & - & $30 \%$ & $15.6 \%$ & $20.0 \%$ & $55.2 \%$ \\
\hline $\begin{array}{l}\text { Significant } \\
\text { POPF }\end{array}$ & $11 \%$ & - & - & - & - & $7.8 \%$ & - & - & $6.3 \%$ & $20.0 \%$ & $13.8 \%$ \\
\hline Morbidity & $5.6 \% C D>2$ & $58.3 \%$ & - & - & $53 \%$ & $53.4 \%$ & - & $95 \%$ & $25.0 \%$ & $40.0 \%$ & $\begin{array}{l}15.5 \% \\
C D>2\end{array}$ \\
\hline $\begin{array}{l}\text { Hospital stay } \\
\text { (days) }\end{array}$ & $6(4-118)$ & $14.9 \pm 6.6$ & - & - & $15(6-53)$ & 6(4-68) & $10.2 \pm 8.5$ & $\begin{array}{l}24.5 \\
(9-311)\end{array}$ & $7(5-52)$ & $9.0(7-20)$ & $\begin{array}{l}14.0 \\
(11.0-17.3)\end{array}$ \\
\hline Readmission & - & - & - & - & - & $22.4 \% 90 d$ & $6.8 \% 30 d$ & - & $6.3 \% 90 d$ & - & - \\
\hline Mortality & $0.9 \%$ 30d & $0 \% 30 d$ & - & $0 \%$ 30d & $0 \%$ 90d & $3.4 \%$ 90d & $6.9 \%$ 90d & $5 \% 90 d$ & $3.1 \%$ 90d & $0 \% 30 d$ & $1.7 \%$ 30d \\
\hline Survival & MS: 25.3 m & $\begin{array}{l}\text { 1,3,5y-DFS:100, } \\
92,83 \% \\
\text { 1,3,5y-OS:100, } \\
92,75 \%\end{array}$ & - & $\begin{array}{l}\text { 5y-OS: } \\
53.6 \%\end{array}$ & - & $\begin{array}{l}\text { 1,2,3,4,5y-OS: } \\
66.5,43.3,43.3 \% \\
38.5,32.1 \%\end{array}$ & $\begin{array}{l}\text { MS: } \\
20.7 \text { m }\end{array}$ & $\begin{array}{l}\text { MS: } \\
35.5 \text { m; } \\
1,3,5 y- \\
\text { DFS: 62.3, } \\
37.9 \\
25.7 \% \\
1,3,5 y-O S: \\
80.5,49.2 \\
39.7 \%\end{array}$ & - & $\begin{array}{l}\text { 1,3,5y-OS: } \\
100,80 \\
67 \%\end{array}$ & MS: $45 \mathrm{~m}$ \\
\hline
\end{tabular}

OS overall survival rate, $M S$ median survival time, $m$ month

revealed the HR of overall survival rate was comparable between LPD and OPD. We agree with the viewpoint of Croome et al. that neither the laparoscopic or open procedure was technically superior would largely depend on the technique of surgeon and on pathologic analytic variability. Thus, a technically similar oncologic resection could be performed regardless of whether the open or laparoscopic approach was meticulously used [14].

Regarding the operative time and blood loss, our pooled data indicated similar outcomes to the previous studies (Table 3). Because of the intricate dissection and complicated gastrointestinal, LPD present a more demanding and challenging approach for pancreatic surgeons. Kendrick and Cusati report their initial duration of LPD to be approximately $8 \mathrm{~h}$, which improved to $5 \mathrm{~h}$ after approximately 50 cases [47]. Therefore, adequate training and optimizing surgical potency to reduce the operative time is required before LPD becomes a generally accepted and sustainable procedure. Another benefit of laparoscopic surgery lies in the enhanced postoperative recovery. Reduced use of analgesic drugs, shortened time of abdominal cavity exposure, and earlier postoperative activities are considered to be the main reasons.

This systematic review and meta-analysis of LDP versus ODP for pancreatic-head and periampullary malignancy represents the most comprehensive collection of evidence available within this field. However, the results should still be explained with caution for several limitations. First, only one study was RCT, while others were NRCTs. Selection biases necessarily consist in surgeons' or patients' decision on operation and adjuvant therapy. Moreover, various biases are real concerns because hardly any of the included studies employed standardized appraisal for the end points. Second, clinical heterogeneity needs significant attention. The surgical techniques, take the different the extension of lymph node dissection for example, and histopathological protocols were variable in both open and laparoscopic groups. Third, there are biological differences between PDAC and PAAC. Although a subgroup analysis was conducted, we should still admit various 
histologies unpowered our outcomes because several studies did not differentiate PDAC and PAAC in their studies, and even in PAAC, there are several different histologies. Last but not least, it should be kept in mind that these studies were on behalf of the best centers' experience on LPD around the world. The literature showed that specialization in pancreatic surgery results in both better short- and long-term survival [48]. Obtained conclusions might not be feasible in less specialized centers.

\section{Conclusions}

Our meta-analysis demonstrated that in contrasted to OPD, LPD could achieve short-term advantages within blood loss, and postoperative morbidity for pancreatic-head and periampullary malignancy. Moreover, both procedures have comparable oncological and long-term survival outcomes. LPD may serve as a promising alternative to OPD in selected individuals suffered pancreatic-head and periampullary malignancy. However, taking into account all the limitations of the study, methodologically high-quality controlled clinical trials are necessary for further evaluation. Anyway, we believe our study could serve as a useful background for future researches.

\section{Abbreviations \\ Cl: Confidence intervals; HR: Hazard ratio; ISGPF: International Study Group for Pancreatic Fistula; LDP: Laparoscopic distal pancreatectomy; LPD: Laparoscopic pancreaticoduodenectomy; MIPD: Minimally invasive pancreaticoduodenectomy; NOS: Newcastle-Ottawa Quality Assessment Scale; NRCT: Nonrandomized comparative study; OPD: Open pancreaticoduodenectomy; OR: Odds ratio; PAAC: Periampullary adenocarcinoma; PD: Pancreaticoduodenectomy; PDAC: Pancreatic duct adenocarcinoma; RCT: Randomized controlled trial; RLN: Retrieved lymph nodes; RPD: Robotic pancreaticoduodenectomy; SD: Standard deviation; SE: Standard error; WMD: Weighted mean difference}

\section{Availability of data and materials}

The datasets analyzed during the current study available from the corresponding author on reasonable request.

\section{Authors' contributions}

KC and YP designed the study; XLL collected relevant literature and conducted the analysis of pooled data; KC and HM wrote the manuscript; XFW proofread and revised the manuscript. All authors have approved the version to be published.

\section{Ethics approval and consent to participate}

Not applicable.

\section{Consent for publication}

Not applicable.

\section{Competing interests}

The authors declare that they have no competing interests.

\section{Publisher's Note}

Springer Nature remains neutral with regard to jurisdictional claims in published maps and institutional affiliations.

\section{Author details}

'Department of General Surgery, Sir Run Run Shaw Hospital, School of Medicine, Zhejiang University, 3 East Qingchun Road, Hangzhou 310016,
Zhejiang Province, China. ${ }^{2}$ School of Medicine, Zhejiang University, 866 Yuhangtang Road, Hangzhou 310058, Zhejiang Province, China.

Received: 3 April 2018 Accepted: 21 June 2018

Published online: 03 July 2018

\section{References}

1. Torre LA, Bray F, Siegel RL, Ferlay J, Lortet-Tieulent J, Jemal A. Global cancer statistics, 2012. CA Cancer J Clin. 2015;65(2):87-108.

2. Rahib L, Smith BD, Aizenberg R, Rosenzweig AB, Fleshman JM, Matrisian LM. Projecting cancer incidence and deaths to 2030: the unexpected burden of thyroid, liver, and pancreas cancers in the United States. Cancer Res. 2014; 74(11):2913-21.

3. Neoptolemos JP, Moore MJ, Cox TF, Valle JW, Palmer DH, McDonald AC, Carter R, Tebbutt NC, Dervenis C, Smith D, et al. Effect of adjuvant chemotherapy with fluorouracil plus folinic acid or gemcitabine vs observation on survival in patients with resected periampullary adenocarcinoma: the ESPAC-3 periampullary cancer randomized trial. Jama. 2012;308(2):147-56.

4. Gawande A. Two hundred years of surgery. N Engl J Med. 2012;366(18):1716-23.

5. Ricci C, Casadei R, Taffurelli G, Toscano F, Pacilio CA, Bogoni S, D'Ambra M, Pagano N, Di Marco MC, Minni F. Laparoscopic versus open distal pancreatectomy for ductal adenocarcinoma: a systematic review and metaanalysis. J Gastrointest Surg. 2015;19(4):770-81.

6. Riviere D, Gurusamy KS, Kooby DA, Vollmer CM, Besselink MG, Davidson BR, van Laarhoven CJ. Laparoscopic versus open distal pancreatectomy for pancreatic cancer. Cochrane Database syst Rev. 2016;4:CD011391.

7. Clavien PA, Barkun J, de Oliveira ML, Vauthey JN, Dindo D, Schulick RD, de Santibanes E, Pekolj J, Slankamenac K, Bassi C, et al. The Clavien-Dindo classification of surgical complications: five-year experience. Ann Surg. 2009; 250(2):187-96.

8. Bassi C, Dervenis C, Butturini G, Fingerhut A, Yeo C, Izbicki J, Neoptolemos J, Sarr M, Traverso W, Buchler M. Postoperative pancreatic fistula: an international study group (ISGPF) definition. Surgery. 2005;138(1):8-13.

9. Wittekind C, Compton C, Quirke P, Nagtegaal I, Merkel S, Hermanek P, Sobin $\mathrm{LH}$. A uniform residual tumor (R) classification: integration of the $\mathrm{R}$ classification and the circumferential margin status. Cancer. 2009;115(15):3483-8.

10. Hozo SP, Djulbegovic B, Hozo I. Estimating the mean and variance from the median, range, and the size of a sample. BMC Med Res Methodol. 2005;5:13.

11. Tierney JF, Stewart LA, Ghersi D, Burdett S, Sydes MR. Practical methods for incorporating summary time-to-event data into meta-analysis. Trials. 2007;8:16.

12. Sharpe SM, Talamonti MS, Wang CE, Prinz RA, Roggin KK, Bentrem DJ, Winchester DJ, Marsh RD, Stocker SJ, Baker MS. Early National Experience with laparoscopic Pancreaticoduodenectomy for ductal adenocarcinoma: a comparison of laparoscopic Pancreaticoduodenectomy and open Pancreaticoduodenectomy from the National Cancer Data Base. J Am Coll Surg. 2015;221(1):175-84.

13. Nussbaum DP, Adam MA, Youngwirth LM, Ganapathi AM, Roman SA, Tyler DS, Sosa JA, Blazer DG 3rd. Minimally invasive Pancreaticoduodenectomy does not improve use or time to initiation of adjuvant chemotherapy for patients with pancreatic adenocarcinoma. Ann Surg Oncol. 2016;23(3):1026-33.

14. Croome KP, Farnell MB, Que FG, Reid-Lombardo KM, Truty MJ, Nagorney DM, Kendrick ML. Total laparoscopic pancreaticoduodenectomy for pancreatic ductal adenocarcinoma: oncologic advantages over open approaches? Ann Surg. 2014;260(4):633-8.

15. Hakeem AR, Verbeke CS, Cairns A, Aldouri A, Smith AM, Menon KV. A matched-pair analysis of laparoscopic versus open pancreaticoduodenectomy: oncological outcomes using Leeds pathology protocol. Hepatobiliary Pancreat Dis Int. 2014;13(4):435-41.

16. Chen S, Chen JZ, Zhan Q, Deng XX, Shen BY, Peng CH, Li HW. Robotassisted laparoscopic versus open pancreaticoduodenectomy: a prospective, matched, mid-term follow-up study. Surg Endosc. 2015;29(12):3698-711.

17. Song KB, Kim SC, Hwang DW, Lee JH, Lee DJ, Lee JW, Park KM, Lee YJ. Matched case-control analysis comparing laparoscopic and open pyloruspreserving Pancreaticoduodenectomy in patients with Periampullary tumors. Ann Surg. 2015;262(1):146-55.

18. Dokmak S, Fteriche FS, Aussilhou B, Bensafta Y, Levy P, Ruszniewski P, Belghiti J, Sauvanet A. Laparoscopic pancreaticoduodenectomy should not be routine for resection of periampullary tumors. J Am Coll Surg. 2015; 220(5):831-8. 
19. Stauffer JA, Coppola A, Villacreses D, Mody K, Johnson E, Li Z, Asbun HJ. Laparoscopic versus open pancreaticoduodenectomy for pancreatic adenocarcinoma: long-term results at a single institution. Surg Endosc. 2017; 31(5):2233-41.

20. Kantor O, Talamonti MS, Sharpe S, Lutfi W, Winchester DJ, Roggin KK, Bentrem DJ, Prinz RA, Baker MS. Laparoscopic pancreaticoduodenectomy for adenocarcinoma provides short-term oncologic outcomes and longterm overall survival rates similar to those for open pancreaticoduodenectomy. Am J Surg. 2017;213(3):512-5

21. Conrad C, Basso V, Passot G, Zorzi D, Li L, Chen HC, Fuks D, Gayet B. Comparable long-term oncologic outcomes of laparoscopic versus open pancreaticoduodenectomy for adenocarcinoma: a propensity score weighting analysis. Surg Endosc. 2017;31(10):3970-8.

22. Palanivelu C, Senthilnathan P, Sabnis SC, Babu NS, Srivatsan Gurumurthy S, Anand Vijai N, Nalankilli VP, Praveen Raj P, Parthasarathy R, Rajapandian S. Randomized clinical trial of laparoscopic versus open pancreatoduodenectomy for periampullary tumours. Br J Surg. 2017;104(11):1443-50.

23. Khaled YS, Fatania K, Barrie J, De Liguori N, Deshpande R, O'Reilly DA, Ammori BJ. Matched case-control comparative study of laparoscopic versus open Pancreaticoduodenectomy for malignant lesions. Surg laparosc Endosc Percutan Tech. 2017;28:47-51.

24. Meng LW, Cai YQ, Li YB, Cai H, Peng B. Comparison of laparoscopic and open Pancreaticoduodenectomy for the treatment of nonpancreatic Periampullary adenocarcinomas. Surg Laparosc Endosc Percutan Tech. 2018;28:56.

25. Gagner M, Pomp A. Laparoscopic pylorus-preserving pancreatoduodenectomy. Surg Endosc. 1994;8(5):408-10.

26. de Rooij T, Klompmaker S, Abu Hilal M, Kendrick ML, Busch OR, Besselink MG. Laparoscopic pancreatic surgery for benign and malignant disease. Nat Rev Gastroenterol Hepatol. 2016;13(4):227-38.

27. Correa-Gallego C, Dinkelspiel HE, Sulimanoff I, Fisher S, Vinuela EF, Kingham TP, Fong Y, DeMatteo RP, D'Angelica MI, Jarnagin WR, et al. Minimallyinvasive vs open pancreaticoduodenectomy: systematic review and metaanalysis. J Am Coll Surg. 2014;218(1):129-39.

28. Lei P, Wei B, Guo W, Wei H. Minimally invasive surgical approach compared with open pancreaticoduodenectomy: a systematic review and metaanalysis on the feasibility and safety. Surg Laparosc Endosc Percutan Tech. 2014;24(4):296-305.

29. Nigri G, Petrucciani N, La Torre M, Magistri P, Valabrega S, Aurello P, Ramacciato G. Duodenopancreatectomy: open or minimally invasive approach? Surgeon. 2014;12(4):227-34.

30. Qin H, Qiu J, Zhao Y, Pan G, Zeng Y. Does minimally-invasive pancreaticoduodenectomy have advantages over its open method? A meta-analysis of retrospective studies. PLoS One. 2014;9(8):e104274.

31. de Rooij T, Lu MZ, Steen MW, Gerhards MF, Dijkgraaf MG, Busch OR, Lips DJ, Festen S, Besselink MG. Minimally invasive versus open

Pancreatoduodenectomy: systematic review and meta-analysis of comparative cohort and registry studies. Ann Surg. 2016;264(2):257-67.

32. Zhang H, Wu X, Zhu F, Shen M, Tian R, Shi C, Wang X, Xiao G, Guo X, Wang M, et al. Systematic review and meta-analysis of minimally invasive versus open approach for pancreaticoduodenectomy. Surg Endosc. 2016;30(12):5173-84.

33. Peng L, Lin S, Li Y, Xiao W. Systematic review and meta-analysis of robotic versus open pancreaticoduodenectomy. Surg Endosc. 2017;31(8):3085-97.

34. Shin SH, Kim YJ, Song KB, Kim SR, Hwang DW, Lee JH, Park KM, Lee YJ, Jun E, Kim SC. Totally laparoscopic or robot-assisted pancreaticoduodenectomy versus open surgery for periampullary neoplasms: separate systematic reviews and meta-analyses. Surg Endosc. 2017;31(9):3459-74.

35. Xiong JJ, Tan CL, Szatmary P, Huang W, Ke NW, Hu WM, Nunes QM, Sutton R, Liu XB. Meta-analysis of pancreaticogastrostomy versus pancreaticojejunostomy after pancreaticoduodenectomy. Br J Surg. 2014; 101(10):1196-208.

36. Zhou W, Lv R, Wang X, Mou Y, Cai X, Herr I. Stapler vs suture closure of pancreatic remnant after distal pancreatectomy: a meta-analysis. Am J Surg. 2010;200(4):529-36.

37. Ecker BL, McMillan MT, Asbun HJ, Ball CG, Bassi C, Beane JD, Behrman SW, Berger AC, Dickson EJ, Bloomston M, et al. Characterization and optimal Management of High-Risk Pancreatic Anastomoses during Pancreatoduodenectomy. Ann Surg. 2017;267:608-16.

38. Sun YL, Zhao YL, Li WQ, Zhu RT, Wang WJ, Li J, Huang S, Ma XX. Total closure of pancreatic section for end-to-side pancreaticojejunostomy decreases incidence of pancreatic fistula in pancreaticoduodenectomy. Hepatobiliary Pancreat Dis Int. 2017;16(3):310-4.
39. Ramana CV, DeBerge MP, Kumar A, Alia CS, Durbin JE, Enelow Rl. Inflammatory impact of IFN-gamma in CD8+ T cell-mediated lung injury is mediated by both Stat1-dependent and -independent pathways. Am J Physiol Lung Cell Mol Physiol. 2015;308(7):L650-7.

40. Howard TJ, Krug JE, Yu J, Zyromski NJ, Schmidt CM, Jacobson LE, Madura $J$ A, Wiebke EA, Lillemoe KD. A margin-negative RO resection accomplished with minimal postoperative complications is the surgeon's contribution to long-term survival in pancreatic cancer. J Gastrointest Surg. 2006;10(10): 1338-45. discussion 1345-1336

41. Demir IE, Jager C, Schlitter AM, Konukiewitz B, Stecher L, Schorn S, Tieftrunk E, Scheufele F, Calavrezos L, Schirren R, et al. R0 versus R1 resection matters after Pancreaticoduodenectomy, and less after distal or Total Pancreatectomy for pancreatic Cancer. Ann Surg. 2017;267:608-16.

42. Youngwirth LM, Nussbaum DP, Thomas S, Adam MA, Blazer DG 3rd, Roman SA, Sosa JA. Nationwide trends and outcomes associated with neoadjuvant therapy in pancreatic cancer: an analysis of 18243 patients. J Surg Oncol. 2017;116(2):127-32.

43. Acharya A, Markar SR, Sodergren MH, Malietzis G, Darzi A, Athanasiou T, Khan AZ. Meta-analysis of adjuvant therapy following curative surgery for periampullary adenocarcinoma. Br J Surg. 2017;104(7):814-22.

44. Luo J, Xiao L, Wu C, Zheng Y, Zhao N. The incidence and survival rate of population-based pancreatic cancer patients: shanghai Cancer registry 20042009. PLoS One. 2013;8(10):e76052.

45. Distler M, Ruckert F, Hunger M, Kersting S, Pilarsky C, Saeger HD, Grutzmann R. Evaluation of survival in patients after pancreatic head resection for ductal adenocarcinoma. BMC Surg. 2013;13:12.

46. Kim K, Chie EK, Jang JY, Kim SW, Oh DY, Im SA, Kim TY, Bang YJ, Ha SW. Role of adjuvant chemoradiotherapy for ampulla of Vater cancer. Int J Radiat Oncol Biol Phys. 2009;75(2):436-41.

47. Kendrick ML, Cusati D. Total laparoscopic pancreaticoduodenectomy: feasibility and outcome in an early experience. Arch Surg. 2010;145(1):19-23.

48. Ahola R, Siiki A, Vasama K, Vornanen M, Sand J, Laukkarinen J. Effect of centralization on long-term survival after resection of pancreatic ductal adenocarcinoma. Br J Surg. 2017;104(11):1532-8.

\section{Ready to submit your research? Choose BMC and benefit from:}

- fast, convenient online submission

- thorough peer review by experienced researchers in your field

- rapid publication on acceptance

- support for research data, including large and complex data types

- gold Open Access which fosters wider collaboration and increased citations

- maximum visibility for your research: over $100 \mathrm{M}$ website views per year

At BMC, research is always in progress.

Learn more biomedcentral.com/submissions 